\title{
The Case for Massive, Evolving Winds in Black Hole X-ray Binaries
}

\author{
Joey Neilsen ${ }^{1, *}$ \\ Boston University Department of Astronomy, 725 Commonwealth Avenue, Room 416D, Boston, MA 02215
}

\begin{abstract}
In the last decade, high-resolution X-ray spectroscopy has revolutionized our understanding of the role of accretion disk winds in black hole X-ray binaries. Here I present a brief review of the state of wind studies in black hole X-ray binaries, focusing on recent arguments that disk winds are not only extremely massive, but also highly variable. I 'show how new and archival observations at high timing and spectral resolution continue to highlight the intricate links between the inner accretion flow, relativistic jets, and accretion disk winds. Finally, I discuss methods to infer the 'driving mechanisms of observed disk winds and their implications for connections between mass accretion and ejection processes.
\end{abstract}

Keywords: accretion, accretion disks; black hole physics; stars: winds, outflows; X-rays: binaries; X-rays: individual (GRO J1655-40)

\section{Introduction}

In the last 20 years, we have seen the discovery of a multitude of highly-ionized absorbers in moderate and high-resolution X-ray spectra of black hole and neutron 'star X-ray binaries (e.g. Ebisawa 1997; Kotani et al. 1997; Brandt \& Schulz 2000; Kotani et al. 2000a,b; Lee et al. 2002; Sidoli et al. 2001, 2002; Schulz \& Brandt 2002; Parmar et al. 2002; Boirin \& Parmar 2003; Boirin et al. 2004, 2005; Miller et al. 2004, 2006a,b, 2008, 2011; Neilsen \& Lee 2009; Neilsen et al. 2011, 2012a; Neilsen \& Homan 2012; Ueda et al. 2004, 2009; Martocchia et al. 2006; Kubota et al. 2007; Blum et al. 2010; Reynolds \& Miller 2010; King et al. 2012b; Díaz Trigo et al. 2006, 2007, 2009, 2012; Diaz Trigo \& Boirin 2012). Often these absorbers are blueshifted, indicative of hot outflowing gas, i.e. accretion 'disk winds. The prevalence of disk winds in X-ray binaries suggests that these outflows may play a crucial role in the physics of accretion and ejection around compact objects. In this brief review, I discuss some recent developments in the influence of ionized disk winds around black holes.

\section{Black Hole Accretion Disk Winds and the Disk- Jet Connection}

Much of the recent work on accretion and ejection processes in black hole outbursts has focused on radio/X-ray correlations (e.g. Gallo et al. 2003; Corbel et al. 2003; Fender \& Belloni 2004; Fender et al. 2004, 2009, although see e.g. Gallo et al. 2012 and references therein for lingering questions about the precise nature of these correlations). Briefly, we now know that typical black hole

\footnotetext{
* Corresponding author

Email address: neilsenj@bu.edu (Joey Neilsen)

${ }^{1}$ Einstein Fellow, Boston University
}

transients emerge from quiescence in X-ray hard states that produce steady, compact jets. They rise in luminosity in this (probably radiatively inefficient; e.g. Esin et al. 1997) hard state, until at some point they undergo a transition towards a much softer state, possibly dominated by a radiatively-efficient disk. This transition has also been associated with major relativistic plasma ejections and the disappearance of steady jets. Eventually, the luminosity falls and they return to quiescence via the hard state.

Over the last decade, this canonical picture of the "diskjet connection" has proved to be a fruitful way to characterize accretion and ejection processes around stellar-mass black holes, and has become the backbone of our understanding of the spectral and timing behavior of black hole transients. But this story cannot be complete, for it fails to describe or account for the presence or the influence of another mode of mass ejection: highly-ionized accretion disk winds, whose behavior in outburst is only now becoming clear.

Just three years after the launch of Chandra, Lee et al. (2002) argued that winds could be associated with the accretion disk, although they were not confined to diskdominated states. Miller et al. (2008) confirmed that in both GRO J1655-40 and GRS 1915+105, absorption lines were stronger in spectrally soft states (see also Neilsen \& Lee 2009). They suggested that higher ionizing flux might be responsible for the changes in the winds, but left open the possibility that other (e.g. geometric) changes might be required as well. Thus it remained unclear how or why winds might change on outburst time scales: were they steady, passive bystanders that simply responded to variations in the ionizing flux, or did they play a role in outbursts, appearing and disappearing just like jets? 

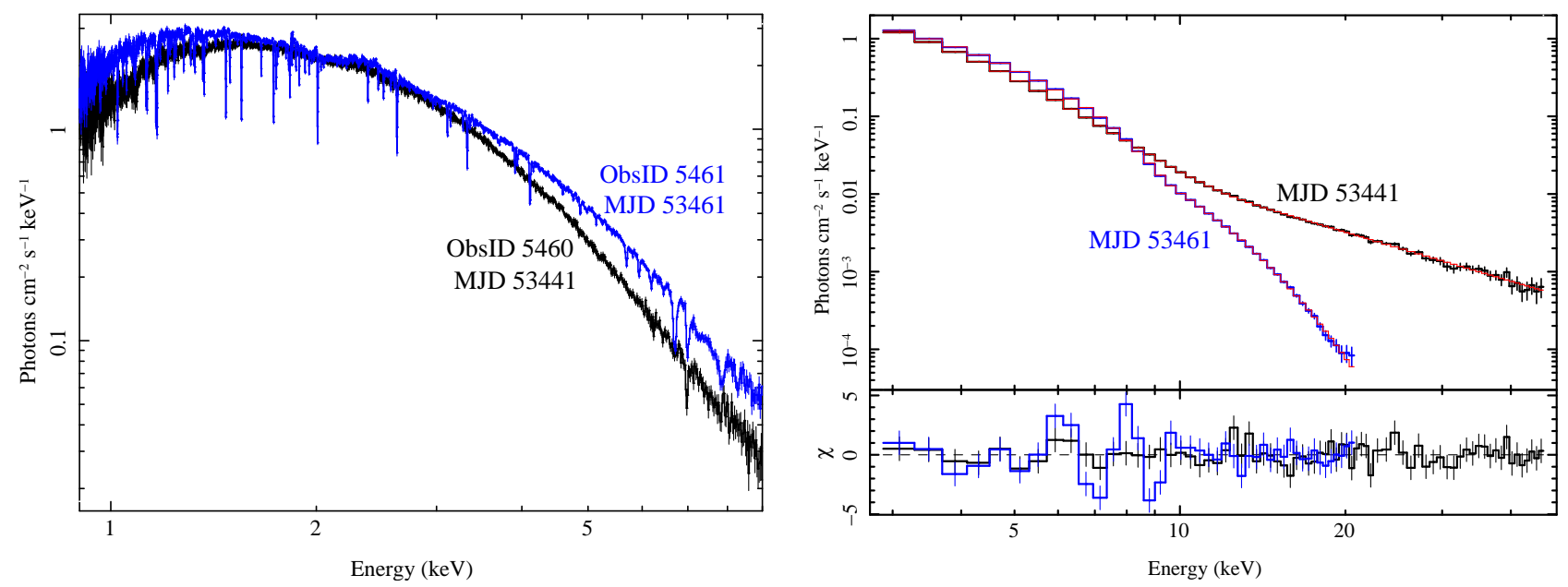

Figure 1: Spectra of GRO J1655-40 from Neilsen \& Homan (2012). (C) 2012. The American Astronomical Society. All rights reserved.) In both panels, black is the spectrum of the hard state and blue is the spectrum of the softer state. Left: Chandra HETGS spectra show only a single line during the first observation, but a rich series of lines from the accretion disk wind in the softer state. Right: $R X T E$ PCA show significant differences in the corresponding broadband X-ray spectra, but we argue (§2.1) that the changes in the ionizing flux cannot explain the differences in the lines.

\subsection{A Case Study in Evolving Winds}

With two high-resolution Chandra HETGS observations of accretion disk winds separated by less than three weeks (Miller et al. 2008; Neilsen \& Homan 2012), the 2005 outburst of the microquasar GRO 1655-40 presents an ideal backdrop against which to test the hypothesis that winds do not evolve during outburst. The Chandra and RXTE spectra are shown in the left and right panels of Figure 1, respectively. The first observation (shown in black) took place during a hard state, while the second observation (shown in blue) occurred during a much softer state. And while the first observation contained an Fe XXVI absorption line near $7 \mathrm{keV}$, the second provided an extremely rich absorption line spectrum that has been studied in great detail (Miller et al. 2006a; Netzer 2006; Miller et al. 2008; Kallman et al. 2009; Neilsen \& Homan 2012; see $\S 3$ for a discussion of the origin of this wind).

Here, let us consider the question: why are the two Chandra absorption line spectra so different? Are the differences driven by changes in the photoionizing flux from the hard state to the soft state, or did the wind physically evolve over those 20 days? Our detailed analysis (Neilsen \& Homan 2012) indicates that the wind must have evolved significantly between the two Chandra observations. This argument can be understood both qualitiatively and quantitatively:

1. A comparison of the hard state and soft state PCA spectra in Figure 1 reveals a clear excess of photons with $E>10 \mathrm{keV}$, which we usually think of as ionizing photons. Thus, at first glance it seems plausible that changes in the ionizing flux could explain the differences in the lines. In fact, however, the ionization of this wind is determined primarily by soft $X$-rays, since many of the visible ions during the softer state, like $\mathrm{O}, \mathrm{Ne}, \mathrm{Na}, \mathrm{Mg}, \mathrm{Al}$, and $\mathrm{Si}$, are effectively transparent to hard X-rays (due to their small cross-sections above $10 \mathrm{keV}$ ). Since the soft Xray spectra of the two observations are quite similar, we conclude that the change in the relevant ionizing flux is negligible and cannot, in and of itself, explain the observed differences in the lines.

2. The physical properties of the rich absorber during the soft state are well known (Miller et al. 2006a, 2008; Kallman et al. 2009), so we can use photoionization codes like XSTAR (Bautista \& Kallman 2001) and the $1 \mathrm{eV}-1 \mathrm{MeV}$ radiation field to generate predictions about ionized absorption during the hard state. Our results (Figure 2; Neilsen \& Homan 2012) clearly indicate that a number of strong absorption lines would have been visible during the hard state if the wind had been steady; the non-detection of these lines confirms that the wind must have evolved during those 20 days. For several simple but realistic scenarios for the geometrical evolution of the wind (Neilsen \& Homan 2012), we argue that the variations in its ionization and column density likely imply an increase in the density and mass loss rate in the wind by a factor between 25 and 300 .

To summarize briefly, after Lee et al. (2002): "ionizing flux is only part of the solution." Based on our careful treatment of photoionization, we find compelling qualitative and quantitative evidence for significant physical changes in the accretion disk wind during the 2005 outburst of GRO J1655-40. In the following section, we argue that broad parallels between this source and other black holes support the conclusion that evolving winds may be an extremely common, if not universal, phenomenon.

\subsection{Ubiquitous, Massive Evolving Winds}

As noted above, Lee et al. (2002) and Miller et al. (2008) pointed out that accretion disk winds seem to be associated with the accretion disk and/or spectrally soft 


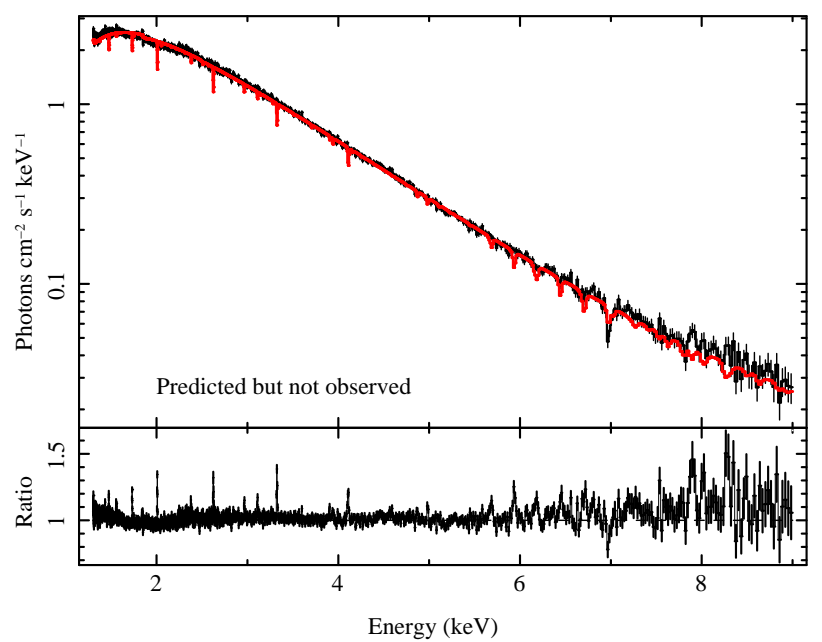

Figure 2: Photoionization models of a steady wind in GRO J1655-40 from Neilsen \& Homan (2012). (C) 2012. The American Astronomical Society. All rights reserved.) Based on Kallman et al. (2009). If the same wind were present in both Chandra HETGS observations, we should have detected a number of strong absorption lines during the hard state. The absence of these lines indicates wind variability over the course of the outburst.

states. Recent work by Ponti et al. (2012) provides convincing evidence for these earlier claims: their archival study of Chandra HETGS, XMM-Newton, and Suzaku observations of stellar mass black holes in outburst demonstrates that accretion disk winds are preferentially detected in softer states ${ }^{2}$. In particular, winds are ubiquitous along the high-luminosity branch of the hardness-intensity diagram after the spectrally hard state (see Lee et al. 2002; Miller et al. 2008; Neilsen \& Homan 2012 for rare cases of weak winds at the high luminosity end of spectrally hard states). In some cases, stringent upper limits have been placed on the existence of hard state winds (e.g. Blum et al. 2010; Miller et al. 2012).

Ponti et al. (2012) suggest that a static absorber with a variable ionization parameter may be unlikely to explain completely the observed behavior of winds in black hole outbursts, although it is also noted that ionization effects may be important and that only detailed photoionization studies can confirm this suggestion. While this is certainly true, black hole wind variability studies on time scales from seconds (Neilsen et al. 2011) to hundreds or thousands of seconds (Lee et al. 2002; Miller et al. 2006b) to weeks and years (Neilsen \& Homan 2012; Blum et al. 2010; Miller et al. 2012) have all required changes in the wind density. It therefore seems likely that the observed outburst behavior of winds will also require such changes, in which case we can conclude that disk winds are preferentially but not

\footnotetext{
${ }^{2}$ Note that disk winds are only detected in systems seen at high orbital inclination, which is consistent with the interpretation that these absorbers are localized near the equatorial plane of the disk. Based on the inclinations of systems with detected disk winds, Ponti et al. estimate a typical wind opening angle $\theta \lesssim 30^{\circ}$. This conclusion echoes established results from studies of neutron star low-mass Xray binaries, where X-ray absorbers are known to be concentrated near the disk plane (e.g. Sidoli et al. 2001; Boirin \& Parmar 2003; Díaz Trigo et al. 2006).
}

exclusively launched at high luminosity, around or after the time the black hole begins to exit the spectrally-hard state.

To test this interpretation, we undertook a Chandra HETGS campaign to catch this phase of a new outburst; the resulting spectra of $4 \mathrm{U} 1630-47$ are shown in Figure 3. The results will be published in detail in future work (Neilsen et al. 2013), but suffice it to say here that with the robust detection of a strong outflow, this campaign was remarkably successful. It should be noted that Kubota et al. (2007) detected a wind in a similar phase of a prior outburst of $4 \mathrm{U} 1630-47$, so our new detection confirms that wind behavior is predictable. We conclude that winds are reliably launched during this outburst phase in black hole X-ray binaries; confirming their absence or weakness in harder and less luminous states will be the subject of future work.

If winds were simply ionized gas along the line of sight, such a conclusion might be interesting but relatively insignificant. In reality, there is now a large and growing body of evidence indicating that disk winds in stellar mass black holes may be extremely massive. In fact, as early as a decade ago, it was discovered that wind mass loss rates $\dot{M}_{\mathrm{w}}$ could be comparable to black hole accretion rates $\dot{M}_{\text {acc }}$ (Lee et al., 2002). More recently Neilsen \& Lee (2009) suggested that radiatively/thermally-driven winds could deplete the mass of the disk enough to suppress relativistic jets. In a few exceptional cases (e.g. the 'heartbeat' state of GRS 1915+105, Neilsen et al. 2011; IGR J170913624, King et al. 2012b), detailed studies have found mass loss rates in excess of $(10-20) M_{\text {acc }}$ ! These remarkable results, too, are supported by the results of Ponti et al. (2012), who find that $\dot{M}_{\mathrm{w}}$ is typically at least twice $\dot{M}_{\text {acc }}$, and approaches $10 \dot{M}_{\mathrm{acc}}$ at high Eddington ratio.

If winds are truly as massive as these results suggest, it begins to seem significant that they are preferentially launched at the same phase of black hole outbursts when we observe the disappearance of steady jets and major changes in the structure of the accretion flow. Could it be that disk winds are indeed the mechanism by which jets are suppressed and state transitions take place, as suggested by Neilsen \& Lee (2009) and Neilsen et al. (2011)? At present, the data cannot rule out this interpretation, but with careful tracking of $\dot{M}_{\text {w }}$ going into this state transition, it may be possible to shed new light on this important question in the near future.

\section{On Inferring Wind Driving Mechanisms}

As noted in $\S 2$, in the last few years there have been a number of developments that suggest deep connections between the X-ray luminosity or accretion rate, the state of the accretion flow, and the behavior of accretion disk winds. The significance of such connections is not entirely clear, however: Miller et al. (2012) (see also Miller et al. 2008) have argued that these some of these connections 

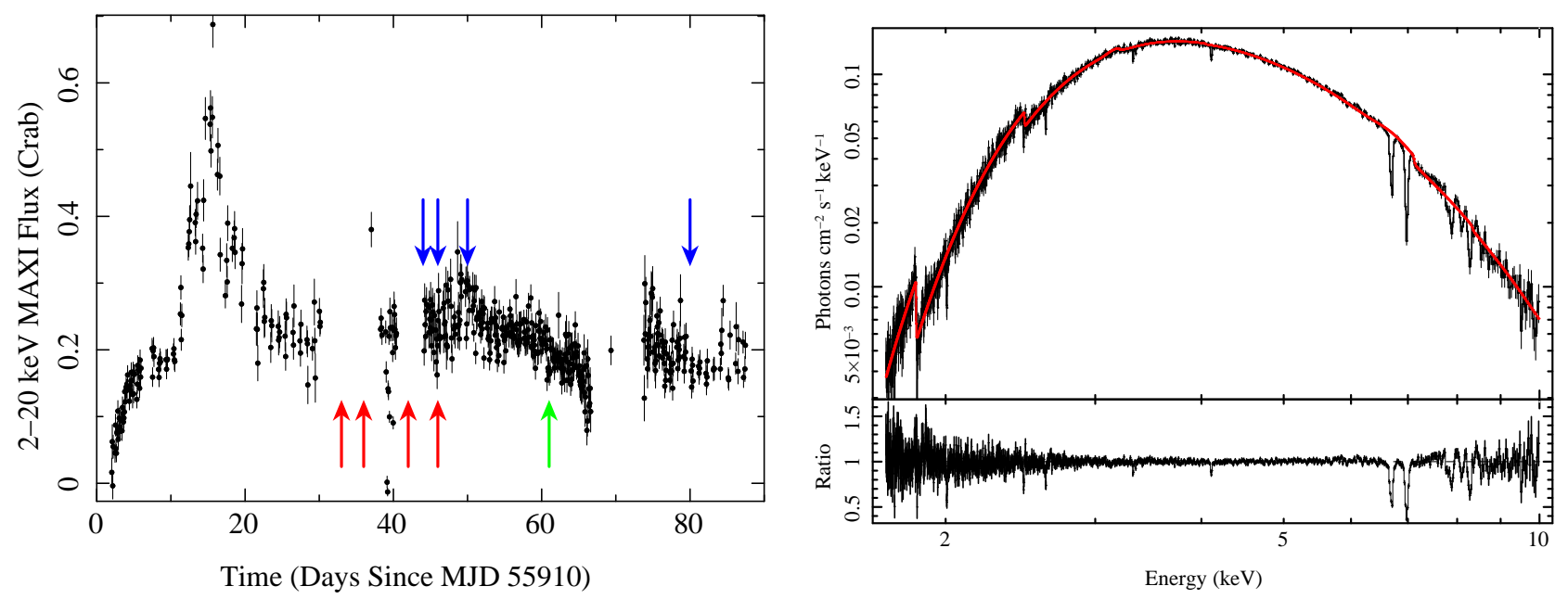

Figure 3: Left: MAXI monitoring of the 2011-2012 outburst of 4U 1630-47, with Chandra HETGS and Suzaku observations indicated by upward arrows, and other wavelengths indicated by downward arrows. Right: Chandra's high-resolution spectra reveal a strong accretion disk wind at precisely the phase of the outburst indicated by Ponti et al. (2012).

can be explained in terms of a magnetic field configuration that changes during outburst, while other authors have presented interpretations based on radiatively- and thermally-driven winds (Neilsen \& Lee 2009; Ueda et al. 2009, 2010; Neilsen et al. 2011, 2012a; Ponti et al. 2012).

Because the detailed implications of these massive evolving winds depend heavily on their formation physics, it is critical to draw robust conclusions about the mechanisms that produce them. To this end, we often take advantage of the fact that the well-known driving mechanisms (radiation pressure, Compton heating, and MHD) typically operate in different regimes of density, ionization, and distance from the black hole (e.g. Proga \& Kallman 2002; Miller et al. 2008).

For example, since radiation pressure is most commonly transmitted via UV resonance absorption lines, it may be ineffective when the gas has little or no opacity in the UV (e.g. at very high ionization, $\xi \gtrsim 10^{3}$ ergs $\mathrm{cm} \mathrm{s}^{-1}$; Proga \& Kallman 2002). In contrast, Compton heating may produce highly-ionized outflows, but because they require a large surface area of gas to be heated to the point that the sound speed exceeds the escape speed, thermally-driven winds are only expected at large distances from the black hole $\left(10^{4}-10^{5} r_{g}\right.$; Begelman et al. 1983; Woods et al. 1996). In addition, simulations at varying luminosities and spectral shapes (e.g. Woods et al. 1996) have shown that thermal driving tends to produce outflows with small mass fluxes and/or gas densities $\left(n \lesssim 10^{12} \mathrm{~cm}^{-3}\right.$ for the specific case of GRO J1655-40; Luketic et al. 2010). MHD processes like the Blandford-Payne mechanism, however, may produce dense outflows at small radii (Blandford \& Payne, 1982).

\subsection{Absorption Lines, Density, and GRO J1655-40}

In principle, then, it is possible to use the observed properties of winds to infer their launching mechanisms.
The difficulty is that many physical factors influence the observability of lines. For example, the X-ray luminosity sets the ionization parameter of the gas:

$$
\xi=\frac{L}{n r^{2}},
$$

while the shape of the ionizing spectrum and the gas density determine which ions are visible at any $\xi$ (Kallman $\&$ Bautista 2001). The density and geometry of the wind determine the equivalent hydrogen column density of the absorber:

$$
N_{\mathrm{H}}=n \Delta r,
$$

and the column density of each individual ion follows from $N_{\mathrm{H}}$, the chemical abundances $A_{i}$, and the ionization balance $x_{i}$ :

$$
N_{i}=x_{i} A_{i} N_{\mathrm{H}}
$$

Finally, the ion column densities are folded into the equivalent width $W_{\lambda}$ of each line via the curve of growth ${ }^{3}$ :

$$
\frac{W_{\lambda}}{\lambda}=\frac{\pi e^{2}}{m_{e} c^{2}} N_{i} \lambda f_{j i}
$$

Here $\lambda$ and $f_{j i}$ are the line wavelength and oscillator strength, respectively. Given the complexity of the connections between the gas properties, the radiation field, and illumination patterns, it is not immediately obvious how any observed wind is produced. However, for a wind that is both very highly ionized $\left(\xi \gtrsim 10^{3} \mathrm{ergs} \mathrm{cm} \mathrm{s}^{-1}\right)$ and sufficiently dense that its implied radius (c.f. Equation 1) is well inside the radius where thermal driving is effective (i.e. the Compton radius, $R<<R_{\mathrm{C}} \sim 10^{11-12} \mathrm{~cm}$ ), the natural conclusion is that MHD processes likely play a role in its launching.

${ }^{3}$ Note that this equation only holds in the optically-thin limit. 
The classic example of this argument in black hole Xray binaries comes from Miller et al. (2006a), who first published the extraordinary Chandra HETGS absorption line spectrum of GRO J1655-40 (Figure 1). Photozioniation analysis indicated a characteristic ionization parameter of $\xi \gtrsim 10^{4} \mathrm{ergs}_{\mathrm{cm} \mathrm{s}}{ }^{-1}$, an order of magnitude too high for line driving to be effective. In addition to many other lines, they detected two FexxII absorption lines at $11.77 \AA$ and $11.92 \AA$ whose ratio can be used as a density diagnostic. Their analysis and subsequent studies (Miller et al. 2008; Kallman et al. 2009) led to the conclusion that the density must have been at least $n \gtrsim 10^{14} \mathrm{~cm}^{-3}$, placing the absorber some three orders of magnitude inside the Compton radius, where thermal driving cannot operate. Based on the high optical depth in the wind and possible saturated lines, Netzer (2006) argued for a lower density $\left(n_{e} \sim 10^{13} \mathrm{~cm}^{-3}\right)$ and ionization parameter $\left(\xi \sim 10^{3}\right)$, which would have implied a more distant wind consistent with thermal driving. Miller et al. (2008) subsequently argued that Netzer's model significantly overpredicted soft X-ray absorption lines, and according to simulations of thermally-driven winds tailored to GRO J1655-40 (Luketic et al. 2010), even the lower density proposed by Netzer (2006) is an order of magnitude too high for thermal driving to be the dominant launching mechanism. By process of elimination, Miller et al. (2006a, 2008); Kallman et al. (2009) concluded that the dense wind in GRO J1655-40 must be powered by magnetic processes ${ }^{4}$.

\subsection{MHD Winds in Black Hole X-ray Binaries?}

But how are we to understand the disk-wind-jet coupling in light of the apparent variations in wind formation physics between different systems? Radiative/thermal driving has been invoked to explain most winds in black hole systems, but there is convincing evidence for an MHD wind in GRO J1655-40. The continuing debate over the accretion disk wind launching mechanism begs the question: is there a single, universal process that governs the interaction between disks, winds, and jets in black hole X-ray binaries? Can the behavior of inflows and outflows be unified in such disparate systems? As our understanding of accretion and ejection physics evolves, it can be instructive to revisit prior observations and the conclusions we draw from them.

Perhaps the most salient development comes from Reynolds (2012), hereafter R12, who demonstrates that the wellknown launching mechanisms (i.e. Compton heating, magnetocentrifugal acceleration, and radiation pressure) can be distinguished not only by the ionization and the density, but also the optical depth of the winds they produce. With a focus on Compton-thick winds, the forbidden regime of

\footnotetext{
${ }^{4}$ This was a long-awaited discovery, particularly since it had been known for some time that both accretion disks and relativistic jets are governed in part by magnetic processes (e.g. Balbus \& Hawley 1991; Blandford \& Znajek 1977; McKinney 2006; Mirabel \& Rodríguez 1999 and references therein).
}

parameter space for each mechanism is clearly set out in terms of the optical depth $\tau$, the Eddington ratio $\lambda$, the ratio $f_{v}$ of the wind's terminal velocity to the escape velocity at the launch radius, and the distance to the black hole $r$ (in units of gravitational radii $r_{g}$ ).

How does this analysis inform the interpretation of the dense, highly-ionized wind in GRO J1655-40, which had a column density $N_{\mathrm{H}} \sim 10^{24} \mathrm{~cm}^{-2}$ (near the Comptonthick limit; Miller et al. 2006a, 2008; Kallman et al. 2009)? Following R12, let us consider each launching mechanism in turn:

1. Thermal Driving: As noted above, and by Miller et al. (2006a, 2008), and Luketic et al. (2010), thermal driving cannot possibly produce such a dense outflow close to the black hole $\left(r \sim(1-7) \times 10^{9} \mathrm{~cm}\right.$ $=1000-6800 r_{g}$; Kallman et al. 2009).

2. Radiative Driving: Again, as argued above following the original work on these data, the wind is far too ionized for UV line driving to be effective at launching the wind. But radiation pressure can also act on free electrons, and R12 shows that in this case, the momentum transferred to the electrons is insufficient to drive a wind if $\lambda<2 f_{v}^{2}$. In other words, the momentum flux in a radiatively-driven wind cannot exceed that of the radiation field.

The soft-state wind of GRO J1655-40 provides an interesting illustration of this constraint. During this particular observation, we estimate the Eddington ratio to be about $\lambda \approx 0.06$ (Neilsen \& Homan 2012). The ratio $f_{v}$ is much harder to determine, since only the line-of-sight velocity can be measured. Kallman et al. (2009) found a blueshift of $\sim 375 \mathrm{~km}$ $\mathrm{s}^{-1}$, which is much less than the escape velocity at plausible launch radii $\left(v_{\text {esc }} \sim 5000-14,000 \mathrm{~km} \mathrm{~s}^{-1}\right.$; $\left.f_{v} \sim 0.03-0.07\right)$. Normally, one might suppose that $f_{v} \sim 1$ and attribute the small blueshift to a velocity primarily perpendicular to the line of sight, but it is difficult to see how this scenario is consistent with the small solid angle of the wind (see below).

If we take the velocities at face value and accept that the wind remains bound to the black hole, we find $\lambda \sim(6-40) \times 2 f_{v}^{2}$, i.e. there is no shortage of momentum in the radiation field. However, this is not a sufficient condition to launch the wind, since the radiation force on the gas is still required to exceed the force of gravity (C. Reynolds 2012, private communication). Thus, despite an abundance of momentum flux, radiation pressure cannot explain the dense, highly-ionized wind in GRO J1655-40 (see also Miller et al. 2006a).

3. Magnetocentrifugal Driving: Although magnetocentrifugal acceleration (e.g. Blandford \& Payne 1982) is not the only MHD process that can drive winds (e.g. Proga 2003; see also Miller et al. 2008 and references therein), it has been studied in great detail 
and lends itself nicely to an analytic constraint on the production of Compton-thick outflows. As shown by R12, Compton-thick magnetocentrifugal winds can only be produced at radii

$$
r<800 \varpi^{-7}\left(\frac{\tau}{\lambda}\right)^{-2}\left(\frac{\Omega}{\pi}\right)^{-2}\left(\frac{\eta}{0.1}\right)^{-2} r_{g},
$$

i.e. where

$$
\frac{\tau}{\lambda}<\sqrt{\frac{800 r_{g}}{\varpi^{7} r}}\left(\frac{\Omega}{\pi}\right)\left(\frac{\eta}{0.1}\right) .
$$

Here $\Omega$ is the solid angle of the wind, $\eta$ is the radiative efficiency of the accretion flow, and $\varpi \sim 2-3$ is the ratio of the size of the acceleration zone of the wind to the launch radius (for more details, see R12 and references therein).

For the dense wind in GRO J1655-40, the observed column density $N_{\mathrm{H}}=10^{24 \pm 0.02} \mathrm{~cm}^{-2}$ implies an optical depth ${ }^{5} \tau \sim 0.67$, from which we estimate $\tau / \lambda \sim 11 \pm 2$. Miller et al. (2006a) use upper limits on emission line strengths to place the tight constraint $\Omega<4 \pi / 9$. If we allow $\varpi \gtrsim 1$ and use the smallest plausible radius $r \sim 970 r_{g}$, Equation 6 for the allowed parameter space for magnetocentrifugal winds becomes $\tau / \lambda<2.0$. That is, given the small solid angle of the wind and its relatively large distance from the black hole, the optical depth of the wind is at least $\sim 5 \times$ too large for it to be launched by magnetocentrifugal processes. However, we stress that other MHD mechanisms (see Miller et al. 2008 and references therein) have not been ruled out.

In short, based on the constraints presented in Reynolds (2012), we find that the dense, highly-ionized wind in GRO J1655-40 cannot be driven by magnetocentrifugal effects. We therefore confirm the original suggestion of Miller et al. (2008) that the wind is driven by some other MHD process, like magnetic pressure (e.g. Proga 2003). It should be noted that R12 uses a simplified model of the wind and driving mechanisms, and that the geometry of observed winds may be somewhat more complicated. For example, as detailed by Giustini \& Proga (2012) and references therein, the bulk properties of winds (e.g. density, ionization, velocity, etc.) may be strong, non-monotonic functions of position, even for outflows with simple streamlines. This is clearly an area where future theoretical work can continue to improve the accuracy and robustness of inferences from observations. In any case, more detailed study of the remarkable wind in GRO J1655-40 is forthcoming.

Finally, we can return to the question at hand: is there a single, universal process that governs the interaction between disks, winds, and jets in black hole X-ray binaries? To the best of our knowledge, we can trace the origin of

\footnotetext{
${ }^{5}$ Since the vast majority of the electrons in an ionized plasma come from hydrogen, for our purposes it is reasonable to assume an ionization fraction very close to 1 .
}

most winds from stellar-mass black holes back to the radiation field of the inner accretion flow (e.g. Lee et al. 2002; Kubota et al. 2007; Neilsen \& Lee 2009; Ueda et al. 2009, 2010; Neilsen et al. 2011, 2012a). This seems to be a compelling argument for the scenario presented in $\S 2$, in which accretion disk winds play an integral role in the evolution of black hole outbursts by virtue of their connections to the radiation field.

However, given that there is one clear case of a magneticallydriven wind, and no hard evidence that other winds are not driven by magnetic fields, this question should still give us pause. Are the results from detailed studies of one wind observed in GRO J1655-40 applicable to the class of black hole binaries as a whole? King et al. (2013) find evidence of a three-way correlation between jet power, wind power, and bolometric luminosity over eight orders of magnitude in black hole mass. This seems to indicate that outflows and inflows could be regulated by a common process related to the mass accretion rate, but the underlying physics of this regulation is still open for discovery.

\section{Conclusions}

In the last few years, a significant effort has been devoted to understanding the physics and behavior of accretion disk winds in black hole X-ray binaries, with important developments coming from both archival studies and new observations. By extrapolating from the specific (exceptional) case of GRO J1655-40 to the ensemble of winds studied by Ponti et al. (2012), we have come to understand that highly-ionized winds are ubiquitous around stellar mass black holes, that they may evolve significantly in outburst, and that they may carry away a significant fraction of the inflowing gas. It is worth reiterating that winds are not necessarily confined to specific states per se, but appear to evolve continuously during outbursts. While their formation physics is complex and challenging to discern, and while MHD processes may be important, it seems that most (but not all) known accretion disk winds are consistent with radiative or thermal driving (Lee et al. 2002; Kubota et al. 2007; Neilsen \& Lee 2009; Ueda et al. 2009, 2010; Neilsen et al. 2011, 2012a; Diaz Trigo \& Boirin 2012 come to a similar conclusion for neutron star binaries).

At once driven by and ionized by the luminosity of the central engine, these massive outflows may require radiation for their existence, but their substantial influence may ultimately be their undoing. By draining vast quantities of mass from the accretion disk, they may not only suppress relativistic jets and cause or facilitate state transitions as seen in GRS 1915+105 (with tentative evidence in other systems; Ponti et al. 2012), but they may also cripple the ability of the disk to launch a wind! In the future, by taking advantage of our ever-growing understanding of the spectral/timing behavior of X-ray binaries in outburst, we will track these evolving, massive, ionized outflows in order 
to continue shedding new light on the physics of accretion and ejection around black holes.

\section{Acknowledgements}

I thank the anonymous referees, whose comments enhanced the context and clarity of the paper, as well as Chris Reynolds and Jon Miller for comments that substantially improved the discussion of wind driving mechanisms. This work was supported by the National Aeronautics and Space Administration through the Smithsonian Astrophysical Observatory contract SV3-73016 to MIT for support of the Chandra X-ray center, which is operated by the Smithsonian Astrophysical Observatory for and on behalf of the National Aeronautics and Space Administration under contract NAS8-03060, and by NASA through the Einstein Fellowship Program, grant PF2-130097.

\section{References}

Balbus, S. A., \& Hawley, J. F. (1991). A powerful local shear instability in weakly magnetized disks. I - Linear analysis. II - Nonlinear evolution. ApJ, 376, 214-233.

Bautista, M. A., \& Kallman, T. R. (2001). The XSTAR Atomic Database. ApJS, 134, 139-149.

Begelman, M. C., McKee, C. F., \& Shields, G. A. (1983). Compton heated winds and coronae above accretion disks. I Dynamics. ApJ, $271,70-88$.

Blandford, R. D., \& Payne, D. G. (1982). Hydromagnetic flows from accretion discs and the production of radio jets. MNRAS, 199, 883-903.

Blandford, R. D., \& Znajek, R. L. (1977). Electromagnetic extraction of energy from Kerr black holes. MNRAS, 179, 433-456.

Blum, J. L., Miller, J. M., \& Cackett, E., et al. (2010). Suzaku Observations of the Black Hole H1743-322 in Outburst. ApJ, 713, 1244-1248.

Boirin, L., Méndez, M., Díaz Trigo, M., et al. (2005). A highlyionized absorber in the X-ray binary $4 \mathrm{U} 1323-62$ : A new explanation for the dipping phenomenon. A\& A, 436, 195-208.

Boirin, L., \& Parmar, A. N. (2003). Discovery of narrow X-ray absorption features from the low-mass X-ray binary X 1254-690 with XMM-Newton. $A \& 3 A, 407,1079-1084$.

Boirin, L., Parmar, A. N., Barret, D., et al. (2004). Discovery of Xray absorption features from the dipping low-mass X-ray binary XB 1916-053 with XMM-Newton. A\&A A 418, 1061-1072.

Brandt, W. N., \& Schulz, N. S. (2000). The Discovery of Broad P Cygni X-Ray Lines from Circinus X-1 with the Chandra HighEnergy Transmission Grating Spectrometer. ApJL, 544, L123L127.

Corbel, S., Nowak, M. A., Fender, R. P., et al. (2003). Radio/Xray correlation in the low/hard state of GX 339-4. A\&SA, 400, $1007-1012$

Diaz Trigo, M., \& Boirin, L. (2012). Disc atmospheres and winds in X-ray binaries. ArXiv e-prints, 1210.0318.

Díaz Trigo, M., Parmar, A. N., Boirin, L., et al. (2006). Spectral changes during dipping in low-mass X-ray binaries due to highlyionized absorbers. A\&A, 445, 179-195.

Díaz Trigo, M., Parmar, A. N., Boirin, L., et al. (2009). Variations in the dip properties of the low-mass X-ray binary XB 1254-690 observed with XMM-Newton and INTEGRAL. A\&A, 493, 145157.

Díaz Trigo, M., Parmar, A. N., Miller, J., et al. (2007). XMMNewton and INTEGRAL spectroscopy of the microquasar GRO J1655-40 during its 2005 outburst. A\&A, 462, 657-666.

Díaz Trigo, M., Sidoli, L., Boirin, L., et al. (2012). XMM-Newton observations of GX $13+1$ : correlation between photoionised absorption and broad line emission. A\&SA, 543, A50-A66.
Ebisawa, K. (1997). X-Ray Spectroscopy of Binary Sources with ASCA - Black Hole Candidates and Super-Soft Sources. In F. Makino \& K. Mitsuda (Ed.), X-Ray Imaging and Spectroscopy of Cosmic Hot Plasmas (pp. 427-431).

Esin, A. A., McClintock, J. E., \& Narayan, R. (1997). Advectiondominated Accretion and the Spectral States of Black Hole X-Ray Binaries: Application to Nova MUSCAE 1991. ApJ, 489, 865889.

Fender, R., \& Belloni, T. (2004). GRS 1915+105 and the Disc-Jet Coupling in Accreting Black Hole Systems. ARA \& A, 42, 317-364.

Fender, R. P., Belloni, T. M., \& Gallo, E. (2004). Towards a unified model for black hole X-ray binary jets. MNRAS, 355, 1105-1118.

Fender, R. P., Homan, J., \& Belloni, T. M. (2009). Jets from black hole X-ray binaries: testing, refining and extending empirical models for the coupling to X-rays. MNRAS, 396, 1370-1382.

Gallo, E., Fender, R. P., \& Pooley, G. G. (2003). A universal radioX-ray correlation in low/hard state black hole binaries. MNRAS, $344,60-72$.

Gallo, E., Miller, B. P., \& Fender, R. (2012). Assessing luminosity correlations via cluster analysis: evidence for dual tracks in the radio/X-ray domain of black hole X-ray binaries. MNRAS, 423, $590-599$.

Giustini, M., \& Proga, D. (2012). On the Diversity and Complexity of Absorption Line Profiles Produced by Outflows in Active Galactic Nuclei. ApJ, 758, 70-79.

Kallman, T., \& Bautista, M. (2001). Photoionization and HighDensity Gas. ApJS, 133, 221-253.

Kallman, T. R., Bautista, M. A., \& Goriely, S., et al. (2009). Spectrum Synthesis Modeling of the X-Ray Spectrum of GRO J1655-40 Taken During the 2005 Outburst. ApJ, 701, 865-884.

King, A. L., Miller, J. M., \& Raymond, J., et al. (2013). Regulation of Black Hole Winds and Jets Across the Mass Scale. ApJ, 762, 103-121

King, A. L., Miller, J. M., \& Raymond, J., et al. (2012a). ApJL, $746, \mathrm{~L} 20-\mathrm{L} 25$.

Kotani, T., Ebisawa, K., \& Dotani, T., et al. (2000a). ASCA Observations of the Absorption Line Features from the Superluminal Jet Source GRS 1915+105. ApJ, 539, 413-423.

Kotani, T., Ebisawa, K., \& Inoue, H., et al. (2000b). Discovery of Iron-K Absorption Lines from the Transient Jets GRS 1915+105 and GRO J1655-40. Advances in Space Research, 25, 445-448.

Kotani, T., Kawai, N., \& Matsuoka, M., et al. (1997). ASCA observations of galactic jet systems. In C. D. Dermer, M. S. Strickman, \& J. D. Kurfess (Ed.), Proceedings of the Fourth Compton Symposium (pp. 922-926). volume 410 of American Institute of Physics Conference Series.

Kubota, A., Dotani, T., Cottam, J., et al. (2007). Suzaku Discovery of Iron Absorption Lines in Outburst Spectra of the X-Ray Transient 4U 1630-472. PASJ, 59, 185-198.

Lee, J. C., Reynolds, C. S., \& Remillard, R., et al. (2002). HighResolution Chandra HETGS and Rossi X-Ray Timing Explorer Observations of GRS 1915+105: A Hot Disk Atmosphere and Cold Gas Enriched in Iron and Silicon. ApJ, 567, 1102-1111.

Luketic, S., Proga, D., \& Kallman, T. R., et al. (2010). On the Properties of Thermal Disk Winds in X-ray Transient Sources: A Case Study of GRO J1655-40. ApJ, 719, 515-522.

Martocchia, A., Matt, G., Belloni, T., et al. (2006). The XMMNewton view of GRS $1915+105 . A \& 3 A, 448,677-687$.

McKinney, J. C. (2006). General relativistic magnetohydrodynamic simulations of the jet formation and large-scale propagation from black hole accretion systems. MNRAS, 368, 1561-1582.

Miller, J. M., Maitra, D., \& Cackett, E. M., et al. (2011). A Fast X-ray Disk Wind in the Transient Pulsar IGR J17480-2446 in Terzan 5. ApJL, 731, L7-L11.

Miller, J. M., Raymond, J., \& Fabian, A., et al. (2006a). The magnetic nature of disk accretion onto black holes. Nature, 441, 953955.

Miller, J. M., Raymond, J., \& Fabian, A. C., et al. (2004). Chandra/High Energy Transmission Grating Spectrometer Spectroscopy of the Galactic Black Hole GX 339-4: A Relativistic Iron Emission Line and Evidence for a Seyfert-like Warm Absorber. 
ApJ, 601, 450-465.

Miller, J. M., Raymond, J., \& Fabian, A. C., et al. (2012). The Disk-wind-Jet Connection in the Black Hole $\mathrm{H}$ 1743-322. ApJL, 759, L6-L9.

Miller, J. M., Raymond, J., \& Homan, J., et al. (2006b). Simultaneous Chandra and RXTE Spectroscopy of the Microquasar H1743322: Clues to Disk Wind and Jet Formation from a Variable Ionized Outflow. ApJ, 646, 394-406.

Miller, J. M., Raymond, J., \& Reynolds, C. S., et al. (2008). The Accretion Disk Wind in the Black Hole GRO J1655-40. ApJ, 680, 1359-1377.

Mirabel, I. F., \& Rodríguez, L. F. (1999). Sources of Relativistic Jets in the Galaxy. ARA\&A, 37, 409-443.

Neilsen, J., \& Homan, J. (2012). A Hybrid Magnetically/Thermally Driven Wind in the Black Hole GRO J1655-40? ApJ, 750, 27-34.

Neilsen, J., \& Lee, J. C. (2009). Accretion disk winds as the jet suppression mechanism in the microquasar GRS 1915+105. Nature, 458, 481-484.

Neilsen, J., Petschek, A. J., \& Lee, J. C. (2012a). Accretion disc wind variability in the states of the microquasar GRS 1915+105. MNRAS, (p. 502-511).

Neilsen, J., Ponti, G., Coriat, M., et al. (2013). Winds, Jets, and State Transitions in the 2011-2012 Outburst of 4U 1630-47. in preparation.

Neilsen, J., Remillard, R. A., \& Lee, J. C. (2011). The Physics of the "Heartbeat" State of GRS 1915+105. ApJ, 737, 69-88.

Netzer, H. (2006). A Thermal Wind Model for the X-Ray Outflow in GRO J1655-40. ApJL, 652, L117-L120.

Parmar, A. N., Oosterbroek, T., Boirin, L., et al. (2002). Discovery of narrow X-ray absorption features from the dipping low-mass Xray binary X 1624-490 with XMM-Newton. A\&A A, 386, 910-915.

Ponti, G., Fender, R. P., \& Begelman, M. C., et al. (2012). Ubiquitous equatorial accretion disc winds in black hole soft states. MNRAS, 422, L11-L15.

Proga, D. (2003). Numerical Simulations of Mass Outflows Driven from Accretion Disks by Radiation and Magnetic Forces. ApJ, 585, 406-417.

Proga, D., \& Kallman, T. R. (2002). On the Role of the Ultraviolet and X-Ray Radiation in Driving a Disk Wind in X-Ray Binaries. ApJ, 565, 455-470.

Reynolds, C. S. (2012). Constraints on Compton-thick Winds from Black Hole Accretion Disks: Can We See the Inner Disk? ApJL, 759, L15-L19.

Reynolds, M. T., \& Miller, J. M. (2010). Chandra Grating Spectroscopy of the Be/X-ray Binary 1A 0535+262. ApJ, 723, 17991805 .

Schulz, N. S., \& Brandt, W. N. (2002). Variability of the X-Ray P Cygni Line Profiles from Circinus X-1 near Zero Phase. ApJ, 572, 971-983.

Sidoli, L., Oosterbroek, T., Parmar, A. N., et al. (2001). An XMMNewton study of the X-ray binary MXB 1659-298 and the discovery of narrow X-ray absorption lines. A\&A, 379, 540-550.

Sidoli, L., Parmar, A. N., Oosterbroek, T., et al. (2002). Discovery of complex narrow X-ray absorption features from the low-mass X-ray binary GX 13+1 with XMM-Newton. A\&A, 385, 940-946.

Ueda, Y., Honda, K., Takahashi, H., et al. (2010). Suzaku Observation of GRS 1915+105: Evolution of Accretion Disk Structure during Limit-cycle Oscillation. ApJ, 713, 257-268.

Ueda, Y., Murakami, H., \& Yamaoka, K., et al. (2004). Chandra High-Resolution Spectroscopy of the Absorption-Line Features in the Low-Mass X-Ray Binary GX 13+1. ApJ, 609, 325-334.

Ueda, Y., Yamaoka, K., \& Remillard, R. (2009). GRS 1915+105 in "Soft State": Nature of Accretion Disk Wind and Origin of X-ray Emission. ApJ, 695, 888-899.

Woods, D. T., Klein, R. I., \& Castor, J. I. (1996). X-Ray-heated Coronae and Winds from Accretion Disks: Time-dependent Twodimensional Hydrodynamics with Adaptive Mesh Refinement. ApJ, 461, 767-804. 